\title{
Reflets
}

Revue ontaroise d'intervention sociale et communautaire

\section{Qui sont les étudiantes du programme de formation francophone d'infirmières praticiennes?}

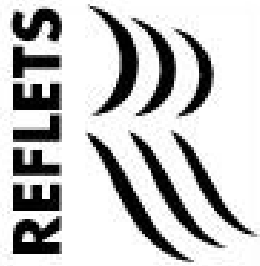

\section{Suzanne Caty, Isabelle Michel, Raymond Pong et Dianne Stewart}

Volume 5, numéro 2, automne 1999

La santé des francophones de l’Ontario

URI : https://id.erudit.org/iderudit/026282ar

DOI : https://doi.org/10.7202/026282ar

Aller au sommaire du numéro

Éditeur(s)

Reflets : Revue ontaroise d'intervention sociale et communautaire

ISSN

1203-4576 (imprimé)

1712-8498 (numérique)

Découvrir la revue

Citer cet article

Caty, S., Michel, I., Pong, R. \& Stewart, D. (1999). Qui sont les étudiantes du programme de formation francophone d'infirmières praticiennes? Reflets, 5(2), 239-246. https://doi.org/10.7202/026282ar

Tous droits réservés (C) Reflets : Revue ontaroise d'intervention sociale et communautaire, 1999
Ce document est protégé par la loi sur le droit d'auteur. L'utilisation des services d’Érudit (y compris la reproduction) est assujettie à sa politique d'utilisation que vous pouvez consulter en ligne.

https://apropos.erudit.org/fr/usagers/politique-dutilisation/ 


\title{
Qui sont les étudiantes $\mathrm{du}$ programme de formation francophone d'infirmières praticiennes? ${ }^{1}$
}

\author{
Suzanne C aty, Inf. M Sc, I sabelle M ichel, Inf. M A , R aymond Pong, \\ PhD et D ianne Stewart, Inf. B ScN ${ }^{2}$
}

\section{Introduction}

En 1994, dans le cadre de la réforme des services de santé, le ministère de la Santé de l'O ntario a lancé le projet d'implantation du rôle des infirmières praticiennes (IP) en O ntario (M inistère de la Santé de I'O ntario 1994). Les infirmières praticiennes sont des infirmières autorisées avec une formation supplémentaire qui leur permet de dispenser desservices de santé intégraux et globaux. C es services sont centrés sur la promotion de la santé, la prévention de la maladie, la guérison, la réadaptation et le soutien. Ils sont inhérents aux soins de santé primaires. Les services offerts par les infirmières praticiennes sont différents, mais complémentairesaux services offerts par les médecins de familles et les autres professionnels de la santé. Les infirmières praticiennes peuvent diagnostiquer certains troubles communs tels l'hypertension, prescrire certains médicaments, demander des tests diagnostic, des échographies ou d'autres tests de laboratoire (Gouvernement de I'O ntario 1998). L'intégration des infirmières praticiennes au sein du secteur des soins primaires permet d'offrir des services de 
haute qualité et d'améliorer l'accès de la population ontarienne aux services de santé (M inistère de la Santé de l'O ntario 1994).

En septembre 1995, le C onseil des programmes universitaires de formation en sciences infirmières de l'O ntario a chargé un consortium d'universités d'élaborer et d'offrir un programme de certificat pour la formation desinfirmières et infirmiers praticiens. C e programme est offert, par la voie de l'éducation à distance, en langue anglaise dans dix universités et en langue française, dans deux universités (Laurentienne et $\mathrm{O}$ ttawa). Le programme de formation IP comprend cinq cours: formation avancée en évaluation de la santé et diagnostic; rôles et responsabilités de l'infirmière ou de l'infirmier praticien; physiopathologie à l'intention de l'infirmière ou de l'infirmier praticien; thérapeutique en soins de santé primaires, stage d'intégration. C e programme à I'intention des infirmières praticiennes est conforme aux normes d'exercice des soins infirmiers émises par l'O rdre des infirmières et infirmiers de l'O ntario.

D eux types d'infirmièresautorisées peuvent obtenir le certificat IP : cellesqui ont un baccalauréat en sciences infirmières et celles qui détiennent un diplôme collégial en sciences infirmières. Ces dernières doivent poursuivre des études menant au baccalauréat en sciences infirmièresavant d'obtenir le certificat IP. Lesétudiantes peuvent étudier à temps plein ou à temps partiel. Entre 1995 et 1998, trois cent soixante- sept (367) étudiantes ont été admises au programme de formation IP et $38(10,4 \%)$ d'entre elles étaient inscrites au programme francophone.

Le $C$ entre de recherche en santé dans les milieux ruraux et du N ord de l'U niversité Laurentienne a obtenu une subvention du Conseil des programmesuniversitaires de formation en sciences infirmières de l'O ntario afin d'entreprendre une étude longitudinale de la population étudiante et des diplômées du certificat IP. Ce projet, effectué au moyen d'un sondage, vise à étudier les caractéristiques des étudiantes et diplômées, leur plan de carrière, leurs opinions sur des sujets tels leurs expériences d'étudiantes et la pratique IP. Le projet a été approuvé par le Comité d'éthique de I'U niversité L aurentienne. 
U n premier sondage a lieu au début du programme. Lors de leur admission au programme, au mois de septembre, un questionnaire est envoyé par la poste à toutes les étudiantes admises. La seule exception à cette directive fut en 1995, année du début du programme, où le questionnaire a été envoyé avec quelques mois de retard. Les résultats du premier questionnaire et certaines informationsobtenueslors de la demande d'admission des premières étudiantes ont servi à peaufiner le questionnaire original et c'est cette version révisée qui depuis lors est utilisée à chaque année.

Cet article, qui analyse les résultats du sondage, a pour objectif de cerner les caractéristiques socio-démographiques de cette population, d'établir leur profil professionnel et de discuter certains éléments relatifs au plan de carrière des étudiantes admises au programme de formation IP francophone entre les années 19951998.Trente-trois (86,8 \%) des38 étudiantes admises au programme francophone ont rempli le questionnaire. Le taux de réponse annuel varie entre $62,5 \%$ et $100 \%$ (C aty, H oule, M ichel, Pong, et Stewart 1996; C aty, M ichel, Pong et Stewart 1997; 1998; 1999).

\section{Caractéristiques sociodémograhiques}

L'âge moyen des trente-deux (97,0 \%) étudiantes francophones étaient de 36,9 ans (étendue: 27 à 47ans). Toutes parlaient couramment le français et $84,8 \%$ parlaient couramment l'anglais. La majorité des infirmières $(81,8 \%)$ était mariée ou vivait en union libre et les deux tiers $(66,7 \%)$ avaient des enfants. D ix-sept $(51,5 \%)$ vivaient dansune communauté du nord-est de la province et douze $(36,4 \%)$ dans une communauté de l'est de la province. D eux vivaient dansle centre-ouest de la province et deux venaient du Q uébec. Les étudiantes étaient inscrites à l'université la plus près de leur domicile (Laurentienne, $n=17 ; 0$ ttawa, $n=16$ ). La majorité $(54,5 \%)$ étudiait à temps partiel.

\section{Caractéristiques professionnelles}

Vingt-quatre $(72,7 \%)$ étudiantes détenaient un baccalauréat en sciences infirmières et neuf $(27,3 \%)$ un diplôme collégial en sciences infirmières. Ces infirmières autorisées avaient 10,5 ans en moyenne d'expérience professionnelle (étendue: 2,5 à 20 ans). 
D urant les cinq années précédant leur admission au programme de formation IP, elles avaient exercé leur profession dans divers organismes de santé. Les deux tiers (66,7\%) avaient travaillé dans un hôpital de soins aigus au sein de départements comme la salle d'urgence, des unités de soins spécialisés et de soins ambulatoires. Plus de la moitié $(63,6 \%)$ avait aussi œuvré dans un milieu communautai re. Cesmilieux comptaient, entre autres, des bureaux de santé publique, des agences de soins à domicile et descliniques spécialisées. Q uelques-unes avaient aussi enseigné au niveau universitaire et collégial. D ans leur plus récent emploi, 93,9\% travaillai ent directement avec les clients. Lorsqu'elles ont été admises au programme de formation IP, 16 (48,5\%) étudiantes travaillaient à temps partiel, 15 (45,4\%) à temps plein et deux $(6,1 \%)$ n'avaient pas d'emploi en soins infirmiers. La majorité $(66,7 \%)$ avait l'intention de continuer à travailler pendant leurs études.

Vingt-quatre $(72,7 \%)$ étudiantes étaient membres d'une association professionnelle reliée aux soinsinfirmiers ou à la santé. Seize d'entre ellesétaient membres de l'A ssociation des infirmières et infirmiers autorisés de l' $\mathrm{O}$ ntario et trois, membres de la $\mathrm{N}$ urse P raditioner A ssodation of 0 ntario. Q uatre ont indiqué qu'ellesétaient affiliées au G roupement desinfirmières et infirmiersfrancophones de l'O ntario.

Les étudiantes ont précisé les raisons pour lesquelles elles voulaient devenir infirmières praticiennes. Les deux principales raisons identifiées par la majorité étaient d'augmenter leur autonomie professionnelle $(63,6 \%)$ et de maximiser leur sphère d'exercice (51,5\%). Le tiers ou plus des répondantes a aussi évoqué les rai sons suivantes: poursuivre leur développement professionnel, offrir des services de santé globaux, atteindre leurs buts personnels et participer à la réforme du système des soins de la santé.

Les étudiantes ont aussi donné leur opinion sur le cheminement académique qu'elle jugeait le plus approprié pour exercer la profession d'infirmière praticienne. D ix-huit (54,5\%) répondantes ont choisi une des deux optionsactuellement offertes par le programme en 0 ntario pour obtenir le certificat IP. En effet, pour 14 d'entre elles (42,4\%), le cheminement idéal devrait 
être l'obtention d'un baccalauréat en sciences infirmières (BSCl nf), suivi du certificat IP. Q uatre (12,1\%) ont opté pour le programme intégré, soit la poursuite simultanée du baccalauréat et du certificat IP. Toutefois, 12 (36,4 \%) ont indiqué que la formation d'infirmière praticienne devrait faire partie d'une maitrise en soins infirmiers et être l'objet d'une forme de concentration.

\section{Plan de carrière}

Les étudiantes ont répondu à plusieurs questions touchant leur plan de carrière en tant qu'infirmières praticiennes. Q uatorze $(42,4 \%)$ répondantes ont affirmé qu'elles avaient l'intention de travailler dansune communauté sous desservie telle que désignée par le ministère de la Santé de I'O ntario. Par contre, le même nombre exprime de l'incertitude à œuvrer dans une telle communauté.

À une question à réponses multiples sur le milieu de pratique, $81,8 \%$ des étudiantes favorisaient un centre de santé communautaire. C ela dit, les autres milieux recevant l'aval d'au moins $15 \%$ des répondantes sont par ordre d'intérêt: bureau de médecin ou service de médecine familiale, bureau de santé publique, clinique de soins spécialisés, bureau de pratique privée, service de soins de santé d'organismes publics (p. ex. université ou collège), salle d'urgence et centre de soins infirmiers dans des régions éloignées. Presque la moitié des répondantes $(45,4 \%)$ voulaient orienter leur pratique d'infirmière praticienne vers les secteurs de la promotion de la santé, de la prévention de la maladie, du traitement de maladies mineures et du maintien de la santé. Parmi celles-ci, 24,2 \% ont répondu qu'elles concentreraient plutôt leur pratique sur la promotion de la santé et un petit nombre (18,2 \%) n'était pas en mesure de préciser le secteur de prédilection. Plus des deux tiers $(69,7 \%)$ voudraient travailler avec des gens de tous les âges et $12,1 \%$ préféreraient travailler surtout avec des adultes. Seulement cinq $(15,2 \%)$ étudiantes avaient un poste d'infirmière praticienne qui les attendaient à la fin de leurs études. 


\section{Conclusion}

Trente-huit (10,4 \%) des 367 infirmières autorisées admises au programme de formation IP durant les dernières quatre années (1995 à 1998) ont étudié en français. D es étudiantes inscrites au programme anglais, vingt et une ont répondu au questionnaire et ont dit parler aussi le français, soit environ $6 \%$ des répondantes (21 sur 329). C e pourcentage est similaire à celui de la population francophone de l'O ntario.

Les 33 étudiantes qui ont participé au sondage étaient des infirmières autorisées qui avaient de nombreuses années d'expérience tant dans les milieux hospitaliers que communautaires. Elles étaient majoritairement mariées, avaient une famille et voulaient continuer à travailler pendant leursétudes. Elles entreprenaient donc leurs études tout en ayant des responsabilités familiales et professionnelles.

L'engagement desétudiantes enversla profession est démontré par le nombre d'étudiantes, membres d'associations professionnelles. Pour elles, devenir infirmière praticienne est un moyen d'augmenter leur autonomie professionnelle et de maximiser leur sphère d'exercice en soinsinfirmiers. Elles préfèrent travailler dans des centres de santé communautaires ou des bureaux de médecins où elles pourraient prodiguer des soins intégraux et globaux à des gens de tout âge. Ces résultats correspondent au but visé par I'initiative du ministère de la Santé de l'O ntario et aux objectifs du programme de formation du certificat IP ( Conseil des programmes universitaires en sciences infirmières de l'O ntario 1995; M inistère de la Santé de l'O ntario 1994).

Le succès de l'intégration des infirmières praticiennes dans le secteur des soins primaires est étroitement lié à une politique gouvernementale qui subventionnera suffisamment de postes IP pour répondre aux besoins de la population et qui utilisera d'une façon innovatrice les services de ces praticiennes. Le ministère de la Santé a été lent à mettre en œuvre cette politique, remettant en question leur engagement initial. Au cours des derniers mois, il y 
a eu déblocage et le ministère de la Santé a annoncé qu'il subventionnera des postes d'infirmières praticiennes dans le secteur des soins communautaires, dans des régions sous desservies et dans les collectivités à risque élevé (G ouvernement de l'O ntario 1998;1999). C es subventions aideront les diplômées à se trouver un poste d'IP et augmenteront I'accessibilité des services de soins primaires à la population ontarienne. II reste à voir maintenant combien de tempsil faudra avant que ces énoncés de politique se transforment en véritables postes d'IP sur le terrain. L'allongement des délais risque de priver la population ontarienne de services en soins primaires dans des régions où elle est déjà défavorisée et de mettre en question une vraie réforme du système de santé.

Les résultats présentés dans cet article proviennent du premier questionnaire de ce projet d'étude longitudinale. Les diplômées du certificat IP ont été ou seront invitées à participer à plusieurs autres sondages : à la sortie du programme, un an après, puis trois ans après I'obtention du certificat IP. Le ministère de la Santé de I'O ntario continuera de subventionner le programme de formation IP jusqu'en I'an 2003 au moins. Le présent projet de recherche continuera d'être financé durant cette même période.

\section{Bibliographie}

CATY, S., L. HOU LE, I. M ICHEL, R.PONG et D. STEWART (1996). The 1995 C ohort of N urse Pracitioner Students: T heir P rofile, V iews, and C areer Plans - E ntry Survey R eport, Sudbury, N orthern $\mathrm{H}$ ealth $\mathrm{H}$ uman R esources R esearch U nit, Laurentian U niversity.

CATY, S., I. M ICHEL, R . PO N G et D. STEWAR T (1997). The 1996 C ohort of N urse Practitioner Students: T heir P rofile, V iews, and C areer Plans - Entry Survey R eport, Sudbury, N orthern H ealth Human R esources R esearch U nit, Laurentian U niversity.

CATY, S., I. M ICHEL, R. PON G et D. ST EWART (1998). T he 1997 C ohort of N urse Pracitioner Students: T heir Profile, V iews, and C areer Plans - Entry Survey R eport, Sudbury, N orthern H ealth H uman R esources R esearch U nit, Laurentian U niversity.

C ATY, S., I. M ICHEL, R. PON G et D. ST EWART (1999). The 1998 C ohort of N urse Practitioner Students: Their P rofile, V iews, and C areer Plans - E ntry Survey R eport, Sudbury, N orthern H ealth Human R esources R esearch U nit, Laurentian U niversity.

CONSEIL DES PROGRAMMES UNIVER SITAIRES EN SCIEN CES IN FIR MIÈRES DE L'O NTAR IO (1995, décembre). L es objectifs du programme infirmières et infirmiers praticiens.

GOVER N M ENT OF ONTAR IO (1998, N ovember 24). H ealth A gencies to R eceive Enhanced $\mathrm{C}$ ommunity $\mathrm{H}$ ealth Funding for $\mathrm{N}$ urse Practitioners, Press $\mathrm{R}$ elease, Toronto. 
GOVER N MENT OF ONTARIO (1999, M arch 15).M ore Funding to Support Increased R ole for $N$ urses, Press $R$ elease, Toronto.

M IN IST ĖRE DE LA SANTÉ DE L'ONTAR IO (1994, décembre). Les infirmières praticennes en $O$ ntario, programme de formation et d' emploi, Toronto.

\section{Notes}

1. Afin d'alléger le texte, le féminin englobe le masculin.

2. Suzanne Caty est professeure titulaire à l'École des sciences infirmières de I'U niversité Laurentienne et est co-chercheure principal e de ce projet. I sabelle M ichel est infirmière clinicienne spécialisée en santé communautaire au Service de santé publique de Sudbury et du district et est co-chercheure de ce projet. R aymond Pong est directeur de recherche du Centre de recherche en santé dans les milieux ruraux et du $\mathrm{N}$ ord de I'U niversité Laurentienne et est co-chercheur principal de ce projet. Dianne Stewart est chercheure associée du C entre de recherche en santé dans les milieux ruraux et du N ord de I'U niversité L aurentienne. 